\title{
Character Association and Path Analysis of Pod Yield and Nutrient Uptake Traits in Groundnut (Arachis hypogaea L.)
}

\author{
Mohammad $\operatorname{Raza}^{1 *}$, M. Reddi Sekhar ${ }^{1}$, M. Shanthi Priya ${ }^{1}$, \\ K.V. Nagamadhuri ${ }^{2}$ and V. Rajarajeswari ${ }^{3}$ \\ ${ }^{1}$ Department of Genetics and Plant Breeding, ${ }^{3}$ Department of Crop Physiology, S.V. \\ Agricultural College, Tirupati, India \\ ${ }^{2}$ Department of Soil Science and Agricultural Chemistry, Institute of Frontier Technology, \\ Tirupati, India \\ *Corresponding author
}

A B S T R A C T

\section{Keywords}

Groundnut,

Correlation, Path analysis, nutrient uptake, Genotype

Article Info

Accepted:

12 February 2018

Available Online:

10 March 2018
Correlation and path analysis was carried with 40 genotypes of groundnut for yield and its component traits. Character association analysis revealed significant and positive association of kernel yield per plant, harvest index, plant height, number of primary branches per plant, seed sulphur uptake, seed iron uptake, seed sulphur (\%) at maturity, seed calcium uptake, number of pods per plant, seed iron (ppm) at maturity, leaf iron (ppm) at 60 DAS and shelling percent with pod yield per plant in groundnut. Path coefficient analysis indicated that kernel yield per plant exerted the highest positive direct effect on pod yield per plant. It was also revealed that plant height, days to maturity, seed calcium uptake and seed iron uptake contributed indirectly to pod yield per plant through kernel yield per plant. These characters also exhibited highly significant and desirable association with pod yield and among themselves. This information could be utilised in formulating a sound selection criterion in groundnut breeding programmes for genetic improvement of high yield potential genotypes with high nutrient uptake ability.

\section{Introduction}

Groundnut (Arachis hypogaea L.) is the World's fourth most important source of edible oil and the third most important source of vegetable protein (Arntez et al., 1994). In India, it occupies an area of $4.68 \mathrm{M}$ ha producing $6.55 \mathrm{Mt}$ with an average productivity of $1400 \mathrm{~kg} \mathrm{ha}^{-1}$ (Anonymous 2015). In groundnut, overall pod yield is constituted by different yield components which make it a quantitatively inherited trait.
So selection of superior genotypes is of paramount importance to improve the yield, to limit the gap of uncertainty in economic yield and to identify nutrient efficient genotypes for nutrient deficient soils which is possible only through the genetic studies of pod yield and component traits. Direct selection of pod yield would not be a reliable approach without giving due importance to its genetic nature, owing to its complex nature of inheritance. Information on the correlation coefficients between the yield components and 
pod yield is a pre-requisite for crop improvement. Though the correlations give information about the component traits, they do not provide a true picture of relative importance of direct and indirect effects of these component traits on pod yield. Hence, the present study was carried out to obtain information on the magnitude of relationship of individual yield components on yield, interrelationships among themselves and to measure their relative importance for pod yield.

\section{Materials and Methods}

The research was conducted at dry the land farm of S. V. Agricultural College, Tirupati, during Kharif 2015. The experimental material consisted of forty genotypes of groundnut. The experiment was laid out in a Randomized Block Design with three replications wherein each genotype was sown in a 5 meter row with a spacing of $30 \mathrm{~cm} \times 10$ $\mathrm{cm}$. All recommended agronomic practices and plant protection measures were adopted in order to exploit the full potential of genotypes. Observations were recorded on randomly chosen five competitive plants in each genotype in each replication for all the 22 characters except for days to $50 \%$ flowering and days to maturity. The data for the latter two characters were recorded on per plot basis. The values of five competitive plants were averaged and expressed as mean of the respective character for that replication. Protein content and oil content in the seed samples were estimated with the help of Universal Grain analyzer (FOSS: Infratech 1241 Grain Analyzer). Sample digestion for nutrient estimation was carried out using Diacid digestion method. Calcium was estimated by Versenate method (Jackson 1967). Estimated of sulphur was done by turbidimetric method (Bhargava, and Raghupati 1993) while, estimation of iron was carried out by using Atomic Absorption
Spectrophotometer (Lindsay and Norvell, 1978). The data were statistically analysed to estimate genotypic and phenotypic correlation coefficients (Johnson et al., 1955) and path coefficient analysis (Dewey and Lu 1959) using Indostat software.

\section{Results and Discussion}

The analysis of variance revealed significant difference among the accessions for all the characters indicating the existence of wide genetic variation among them. The phenotypic and genotypic correlations amongst the characters showed similar trend of association between the character pairs, the latter values being higher in most cases, indicating the preponderance of genetic variation in expression of these traits (Table 1). The characters kernel yield per plant, harvest index, plant height, number of primary branches per plant, seed sulphur uptake, seed iron uptake, seed sulphur (\%) at maturity, seed calcium uptake, number of pods per plant, seed iron (ppm) at maturity, leaf iron (ppm) at 60 DAS and shelling percent showed highly significant positive association with pod yield per plant indicating the positive linear relationship of these characters with pod yield. This report of significant, positive association of pod yield per plant with kernel yield per plant was in accordance with the reports of Kumar et al., (2013). Similar kind of significant positive association of harvest index with pod yield per plant was reported by Sharma and Gupta (2008). The observed positive associations of number of pods per plant and number of branches per plant with pod yield per plant were in conformity with the result of Amarsinghe et al., (2016). Similarly, the report of Nadaf et al., (2011) is in agreement of the significant association of leaf iron content with pod yield per plant. Similar kind of association of pod yield per plant with leaf sulphur (\%) was reported by Ramesh (2006). 
Table.1 Phenotypic $\left(r_{p}\right)$ and genotypic $\left(r_{g}\right)$ correlation coefficients among pod yield and its component traits in 40 genotypes of groundnut

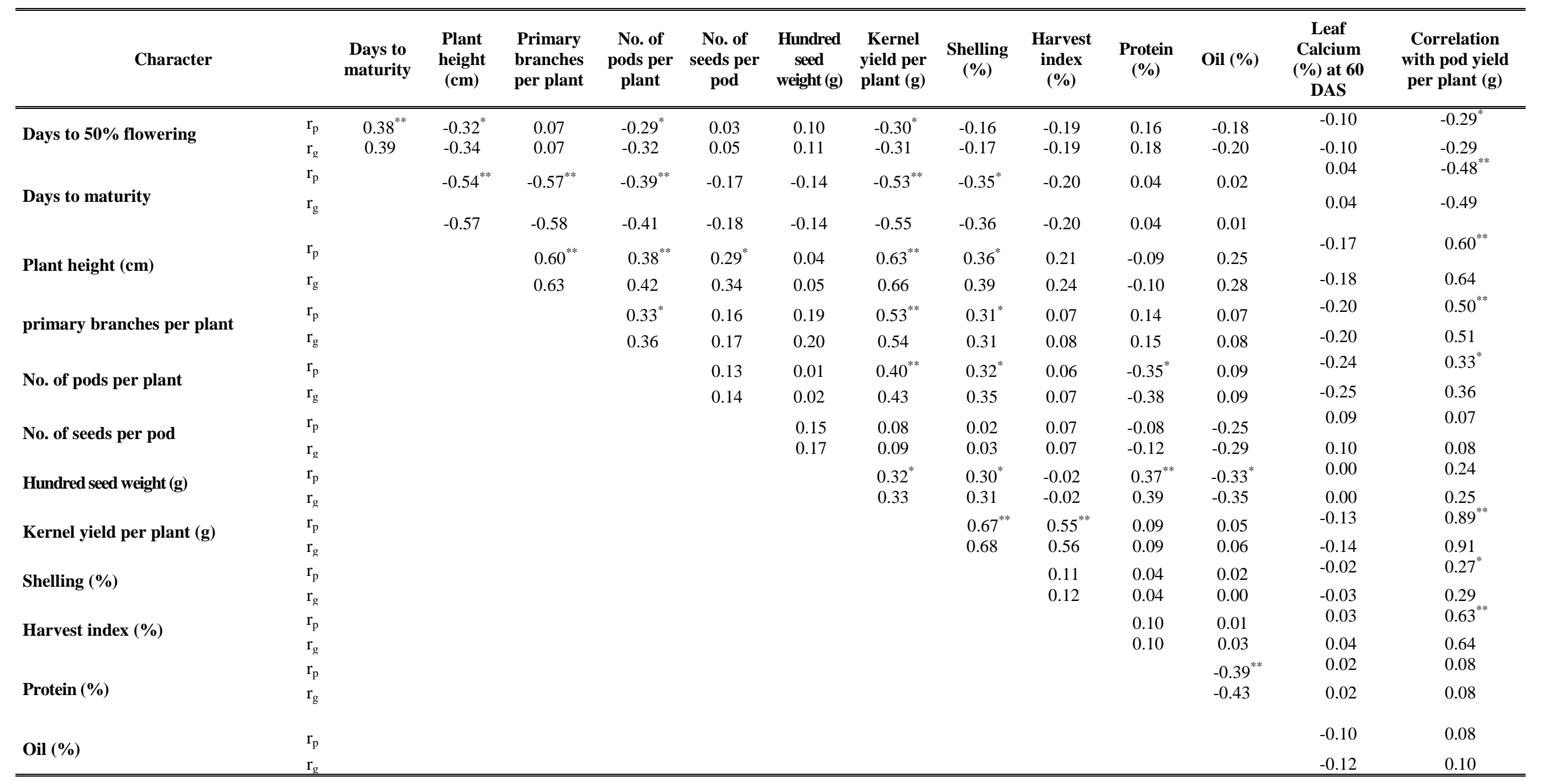

"Significant at $5 \%$ level; ${ }^{* *}$ Significant at $1 \%$ level contd... 
Table 1. Contd...

\begin{tabular}{|c|c|c|c|c|c|c|c|c|c|c|}
\hline Character & & 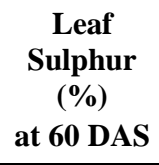 & $\begin{array}{c}\text { Leaf Iron } \\
(\text { ppm) } \\
\text { at } 60 \text { DAS }\end{array}$ & $\begin{array}{c}\text { Seed Calcium } \\
(\%) \\
\text { at maturity }\end{array}$ & $\begin{array}{c}\text { Seed } \\
\text { Sulphur } \\
(\%) \\
\text { at maturity }\end{array}$ & $\begin{array}{c}\text { Seed Iron } \\
(\mathbf{p p m}) \\
\text { at } \\
\text { maturity }\end{array}$ & $\begin{array}{c}\text { Seed calcium } \\
\text { uptake } \\
\left(\mathrm{Kg} \mathrm{ha}^{-1}\right)\end{array}$ & $\begin{array}{c}\text { Seed sulphur } \\
\text { uptake } \\
\left(\mathrm{Kg} \mathrm{ha}^{-1}\right)\end{array}$ & $\begin{array}{c}\text { Seed iron } \\
\text { uptake } \\
\left(\mathrm{Kg} \mathrm{ha}^{-1}\right)\end{array}$ & $\begin{array}{l}\text { Correlation } \\
\text { with pod yield } \\
\text { per plant (g) }\end{array}$ \\
\hline \multirow{2}{*}{ Days to $50 \%$ flowering } & $r_{p}$ & -0.07 & -0.22 & 0.09 & 0.15 & 0.11 & -0.09 & -0.09 & -0.07 & $-0.29^{*}$ \\
\hline & $r_{g}$ & -0.06 & -0.23 & 0.10 & 0.16 & 0.11 & -0.10 & -0.09 & -0.08 & -0.29 \\
\hline \multirow{2}{*}{ Days to maturity } & $\mathrm{r}_{\mathrm{p}}$ & -0.07 & -0.17 & 0.04 & 0.03 & -0.23 & -0.25 & $-0.32^{*}$ & $-0.41^{* *}$ & $-0.48^{* * *}$ \\
\hline & $r_{g}$ & -0.07 & -0.17 & 0.03 & 0.02 & -0.24 & -0.26 & -0.33 & -0.42 & -0.49 \\
\hline \multirow{2}{*}{ Plant height (cm) } & $\mathrm{r}_{\mathrm{p}}$ & 0.08 & 0.19 & -0.15 & 0.10 & $0.34^{*}$ & 0.15 & $0.33^{*}$ & $0.46^{* *}$ & $0.60^{* *}$ \\
\hline & $r_{g}$ & 0.08 & 0.20 & -0.16 & 0.11 & 0.36 & 0.16 & 0.35 & 0.48 & 0.64 \\
\hline \multirow{2}{*}{ primary branches per plant } & $r_{p}$ & -0.01 & 0.03 & 0.15 & 0.02 & 0.25 & 0.15 & 0.14 & $0.30^{*}$ & $0.50^{* *}$ \\
\hline & $r_{g}$ & -0.01 & 0.03 & 0.16 & 0.02 & 0.25 & 0.16 & 0.14 & 0.30 & 0.51 \\
\hline \multirow{2}{*}{ No. of pods per plant } & $r_{p}$ & -0.01 & 0.20 & 0.12 & 0.00 & -0.25 & $0.26^{*}$ & $0.26^{*}$ & 0.04 & $0.33^{*}$ \\
\hline & $r_{g}$ & 0.00 & 0.21 & 0.12 & 0.00 & -0.26 & 0.27 & 0.28 & 0.04 & 0.36 \\
\hline No. of seeds per pod & $r_{g}$ & 0.24 & 0.00 & -0.10 & 0.07 & 0.04 & 0.12 & 0.22 & 0.18 & 0.08 \\
\hline \multirow{2}{*}{ Hundred seed weight (g) } & $r_{p}$ & 0.04 & 0.07 & 0.25 & 0.02 & -0.02 & 0.23 & 0.10 & 0.05 & 0.24 \\
\hline & $r_{g}$ & 0.05 & 0.07 & 0.26 & 0.02 & -0.02 & 0.23 & 0.10 & 0.05 & 0.25 \\
\hline \multirow{2}{*}{ Kernel yield (g) } & $r_{p}$ & 0.18 & $0.29^{*}$ & 0.20 & 0.24 & $0.28^{*}$ & $0.40^{* *}$ & $0.47^{* *}$ & $0.48^{* *}$ & $0.89^{* *}$ \\
\hline & $r_{g}$ & 0.18 & 0.30 & 0.21 & 0.25 & 0.28 & 0.40 & 0.47 & 0.49 & 0.91 \\
\hline \multirow{2}{*}{ Shelling (\%) } & $r_{p}$ & 0.12 & 0.15 & 0.14 & -0.14 & 0.05 & 0.25 & 0.16 & 0.19 & $0.27^{*}$ \\
\hline & $r_{g}$ & 0.12 & 0.16 & 0.14 & -0.14 & 0.05 & 0.25 & 0.16 & 0.19 & 0.29 \\
\hline \multirow{2}{*}{ Harvest index (\%) } & $r_{p}$ & 0.12 & 0.03 & 0.07 & $0.40^{* *}$ & 0.10 & $0.30^{*}$ & $0.48^{* *}$ & $0.32^{*}$ & $0.63^{* *}$ \\
\hline & $r_{g}$ & 0.13 & 0.03 & 0.08 & 0.41 & 0.11 & 0.30 & 0.49 & 0.33 & 0.64 \\
\hline \multirow{2}{*}{ Protein $(\%)$} & $r_{p}$ & 0.06 & 0.08 & 0.12 & 0.22 & 0.01 & 0.02 & 0.09 & 0.03 & 0.08 \\
\hline & $r_{g}$ & 0.07 & 0.08 & 0.12 & 0.23 & 0.01 & 0.02 & 0.09 & 0.03 & 0.07 \\
\hline \multirow{2}{*}{ Oil (\%) } & $r_{p}$ & -0.08 & 0.06 & -0.06 & -0.01 & 0.13 & 0.06 & 0.04 & 0.12 & 0.08 \\
\hline & $r_{g}$ & -0.10 & 0.06 & -0.06 & -0.02 & 0.14 & 0.07 & 0.04 & 0.13 & 0.10 \\
\hline
\end{tabular}

${ }^{*}$ Significant at 5\% level; ${ }^{* *}$ Significant at $1 \%$ level Contd... 
Table 1. Contd...

\begin{tabular}{|c|c|c|c|c|c|c|c|c|c|c|}
\hline Character & & $\begin{array}{c}\text { Leaf } \\
\text { Sulphur }(\%) \\
\text { at } 60 \text { DAS }\end{array}$ & $\begin{array}{c}\text { Leaf Iron } \\
(\mathrm{ppm}) \\
\text { at } 60 \text { DAS }\end{array}$ & $\begin{array}{c}\text { Seed } \\
\text { Calcium } \\
(\%) \\
\text { at maturity }\end{array}$ & $\begin{array}{c}\text { Seed } \\
\text { Sulphur }(\%) \\
\text { at maturity }\end{array}$ & $\begin{array}{l}\text { Seed Iron } \\
\quad(\mathbf{p p m}) \\
\text { at maturity }\end{array}$ & $\begin{array}{c}\text { Seed } \\
\text { calcium } \\
\text { uptake } \\
\left(\mathrm{Kg} \mathrm{ha}^{-1}\right)\end{array}$ & $\begin{array}{c}\text { Seed } \\
\text { sulphur } \\
\text { uptake } \\
\left(\mathrm{Kg} \mathrm{ha}^{-1}\right)\end{array}$ & $\begin{array}{c}\text { Seed iron } \\
\text { uptake } \\
\left(\mathrm{Kg} \mathrm{ha}^{-1}\right)\end{array}$ & $\begin{array}{c}\text { Correlation } \\
\text { with pod yield } \\
\text { per plant (g) }\end{array}$ \\
\hline \multirow{2}{*}{ Leaf Calcium (\%) at 60 DAS } & $r_{p}$ & 0.18 & 0.21 & -0.14 & 0.12 & 0.09 & 0.09 & 0.18 & 0.18 & -0.20 \\
\hline & $r_{g}$ & 0.18 & 0.21 & -0.14 & 0.12 & 0.09 & 0.09 & 0.18 & 0.19 & -0.20 \\
\hline \multirow{2}{*}{ Leaf Sulphur (\%) at 60 DAS } & $r_{p}$ & & 0.14 & -0.03 & 0.14 & 0.17 & 0.11 & 0.24 & 0.25 & 0.13 \\
\hline & $r_{g}$ & & 0.15 & -0.03 & 0.14 & 0.17 & 0.11 & 0.24 & 0.25 & 0.13 \\
\hline \multirow{2}{*}{ Leaf Iron (ppm) at 60 DAS } & $\mathrm{r}_{\mathrm{p}}$ & & & -0.16 & $0.28^{*}$ & -0.07 & 0.16 & $0.36^{*}$ & 0.18 & $0.29^{*}$ \\
\hline & $r_{g}$ & & & -0.16 & 0.29 & -0.07 & 0.16 & 0.36 & 0.18 & 0.29 \\
\hline \multirow{2}{*}{ Seed Calcium (\%) at maturity } & $\mathrm{r}_{\mathrm{p}}$ & & & & $0.31^{*}$ & 0.02 & $0.61^{* *}$ & 0.16 & 0.04 & 0.17 \\
\hline & $\mathrm{r}_{\mathrm{g}}$ & & & & 0.31 & 0.02 & 0.61 & 0.16 & 0.04 & 0.17 \\
\hline \multirow{2}{*}{ Seed Sulphur (\%) at maturity } & $\mathrm{r}_{\mathrm{p}}$ & & & & & 0.20 & $0.35^{*}$ & $0.61^{* *}$ & $0.29^{*}$ & $0.38^{* *}$ \\
\hline & $r_{g}$ & & & & & 0.20 & 0.35 & 0.61 & 0.30 & 0.39 \\
\hline \multirow{2}{*}{ Seed Iron (ppm) at maturity } & $r_{p}$ & & & & & & 0.17 & $0.27^{*}$ & $0.76^{* *}$ & $0.30^{*}$ \\
\hline & $r_{g}$ & & & & & & 0.17 & 0.27 & 0.76 & 0.31 \\
\hline \multirow{2}{*}{ Seed calcium uptake $\left(\mathrm{Kg} \mathrm{ha}^{-1}\right)$} & $\mathrm{r}_{\mathrm{p}}$ & & & & & & & $0.79^{* * *}$ & $0.61^{* *}$ & $0.36^{*}$ \\
\hline & $\mathrm{r}_{\mathrm{g}}$ & & & & & & & 0.79 & 0.61 & 0.37 \\
\hline \multirow{2}{*}{ Seed sulphur uptake (Kg ha $\left.{ }^{-1}\right)$} & $\mathrm{r}_{\mathrm{p}}$ & & & & & & & & $0.75^{* *}$ & $0.50^{* * *}$ \\
\hline & $r_{g}$ & & & & & & & & 0.75 & 0.51 \\
\hline \multirow[t]{2}{*}{ Seed iron uptake $\left(\mathrm{Kg} \mathrm{ha}^{-1}\right)$} & $r_{p}$ & & & & & & & & & $0.49^{* *}$ \\
\hline & $r_{g}$ & & & & & & & & & 0.50 \\
\hline
\end{tabular}

${ }^{\text {*Significant at 5\% level; }{ }^{* *} \text { Significant at } 1 \% \text { level }}$ 
Table.2 Phenotypic (P) and genotypic (G) path coefficients among pod yield per plant and its components in 40 genotypes of Groundnut

\begin{tabular}{|c|c|c|c|c|c|c|c|c|c|c|c|c|c|c|c|c|}
\hline Character & & $\begin{array}{l}\text { Days to } \\
50 \% \\
\text { floweri } \\
\text { ng }\end{array}$ & $\begin{array}{c}\text { Days to } \\
\text { maturit } \\
y\end{array}$ & $\begin{array}{c}\text { Plant } \\
\text { height } \\
(\mathrm{cm})\end{array}$ & $\begin{array}{c}\text { No. of } \\
\text { branches } \\
\text { per plant }\end{array}$ & $\begin{array}{l}\text { No. of } \\
\text { pods per } \\
\text { plant }\end{array}$ & $\begin{array}{l}\text { Kernel } \\
\text { yield per } \\
\text { plant }(g)\end{array}$ & $\begin{array}{c}\text { Shelling } \\
(\%)\end{array}$ & $\begin{array}{c}\text { Harvest } \\
\text { index } \\
(\%)\end{array}$ & $\begin{array}{c}\text { Leaf Iron } \\
(\text { ppm) } \\
\text { at } 60 \mathrm{DAS}\end{array}$ & $\begin{array}{c}\text { Seed } \\
\text { sulphur }(\%) \\
\text { at maturity }\end{array}$ & $\begin{array}{l}\text { Seed iron } \\
\quad(\mathbf{p p m}) \\
\text { at maturity }\end{array}$ & $\begin{array}{c}\text { Seed } \\
\text { calcium } \\
\text { uptake } \\
\left(\mathrm{Kg} \mathrm{ha}^{-1}\right)\end{array}$ & $\begin{array}{c}\text { Seed } \\
\text { sulphur } \\
\text { uptake } \\
\left(\mathrm{Kg} \mathrm{ha}^{-1}\right)\end{array}$ & $\begin{array}{c}\text { Seed iron } \\
\text { uptake } \\
\left(\mathrm{Kg} \mathrm{ha}^{-1}\right)\end{array}$ & $\begin{array}{l}\text { Correlation } \\
\text { with pod } \\
\text { yield (g) }\end{array}$ \\
\hline \multirow{2}{*}{ Days to $50 \%$ flowering } & $\mathrm{P}$ & 0.044 & -0.007 & -0.011 & -0.003 & 0.004 & -0.413 & 0.103 & 0.005 & 0.001 & -0.003 & -0.007 & -0.001 & 0.002 & -0.002 & $-0.289^{*}$ \\
\hline & G & 0.142 & -0.026 & -0.037 & -0.015 & 0.011 & -0.509 & 0.131 & 0.026 & 0.010 & -0.006 & -0.018 & -0.004 & 0.008 & -0.006 & -0.293 \\
\hline \multirow{2}{*}{ Days to maturity } & $\mathrm{P}$ & 0.017 & -0.018 & -0.019 & 0.027 & 0.005 & -0.724 & 0.222 & 0.005 & 0.001 & -0.001 & 0.015 & -0.003 & 0.006 & -0.009 & $-0.477^{* *}$ \\
\hline & G & 0.056 & -0.066 & -0.063 & 0.117 & 0.014 & -0.892 & 0.284 & 0.028 & 0.007 & -0.001 & 0.040 & -0.011 & 0.031 & -0.035 & -0.490 \\
\hline \multirow{2}{*}{ Plant height (cm) } & $\mathrm{P}$ & -0.014 & 0.010 & 0.035 & -0.028 & -0.005 & 0.855 & -0.229 & -0.006 & -0.001 & -0.002 & -0.021 & 0.002 & -0.006 & 0.010 & $0.600^{* *}$ \\
\hline & G & -0.049 & 0.038 & 0.110 & -0.127 & -0.014 & 1.076 & -0.304 & -0.033 & -0.009 & -0.004 & -0.061 & 0.007 & -0.033 & 0.040 & 0.638 \\
\hline \multirow{2}{*}{ No. of branches per plant } & $\mathrm{P}$ & 0.003 & 0.010 & 0.021 & -0.047 & -0.004 & 0.719 & -0.193 & -0.002 & 0.000 & 0.000 & -0.016 & 0.002 & -0.003 & 0.006 & $0.497^{* *}$ \\
\hline & $\mathrm{G}$ & 0.011 & 0.038 & 0.069 & -0.202 & -0.012 & 0.886 & -0.246 & -0.010 & -0.001 & -0.001 & -0.043 & 0.007 & -0.014 & 0.025 & 0.507 \\
\hline \multirow{2}{*}{ No. of pods per plant } & $\mathrm{P}$ & -0.013 & 0.007 & 0.013 & -0.016 & -0.013 & 0.541 & -0.200 & -0.002 & -0.001 & 0.000 & 0.016 & 0.003 & -0.005 & 0.001 & $0.333^{*}$ \\
\hline & G & -0.045 & 0.027 & 0.046 & -0.072 & -0.033 & 0.701 & -0.277 & -0.010 & -0.009 & 0.000 & 0.045 & 0.011 & -0.026 & 0.004 & 0.363 \\
\hline \multirow{2}{*}{ Kernel yield per plant (g) } & $\mathrm{P}$ & -0.013 & 0.010 & 0.022 & -0.025 & -0.005 & 1.356 & -0.421 & -0.015 & -0.001 & -0.005 & -0.018 & 0.004 & -0.008 & 0.010 & $0.891^{* *}$ \\
\hline & G & -0.044 & 0.036 & 0.072 & -0.109 & -0.014 & 1.637 & -0.533 & -0.078 & -0.013 & -0.008 & -0.048 & 0.017 & -0.045 & 0.041 & 0.910 \\
\hline \multirow{2}{*}{ Shelling (\%) } & $\mathrm{P}$ & -0.007 & 0.006 & 0.013 & -0.015 & -0.004 & 0.908 & -0.629 & -0.003 & -0.001 & 0.003 & -0.003 & 0.003 & -0.003 & 0.004 & $0.273^{*}$ \\
\hline & G & -0.024 & 0.024 & 0.043 & -0.064 & -0.012 & 1.116 & -0.782 & -0.016 & -0.007 & 0.005 & -0.008 & 0.011 & -0.015 & 0.016 & 0.288 \\
\hline \multirow{2}{*}{ Harvest index (\%) } & $\mathrm{P}$ & -0.008 & 0.004 & 0.007 & -0.003 & -0.001 & 0.741 & -0.071 & -0.027 & 0.000 & -0.008 & -0.007 & 0.003 & -0.008 & 0.007 & $0.628^{* *}$ \\
\hline & G & -0.027 & 0.014 & 0.026 & -0.015 & -0.002 & 0.913 & -0.092 & -0.139 & -0.001 & -0.014 & -0.018 & 0.013 & -0.046 & 0.027 & 0.638 \\
\hline \multirow{4}{*}{$\begin{array}{l}\text { Leaf Iron (ppm) at } 60 \\
\text { DAS } \\
\text { Seed sulphur (\%) at } \\
\text { maturity }\end{array}$} & $\mathrm{P}$ & -0.010 & 0.003 & 0.007 & -0.001 & -0.003 & 0.397 & -0.096 & -0.001 & -0.005 & -0.006 & 0.005 & 0.002 & -0.006 & 0.004 & $0.291^{*}$ \\
\hline & G & -0.032 & 0.011 & 0.022 & -0.006 & -0.007 & 0.484 & -0.121 & -0.004 & -0.043 & -0.010 & 0.013 & 0.007 & -0.034 & 0.015 & 0.295 \\
\hline & $\mathrm{P}$ & 0.007 & -0.001 & 0.004 & -0.001 & 0.000 & 0.328 & 0.086 & -0.011 & -0.001 & -0.021 & -0.013 & 0.004 & -0.011 & 0.006 & $0.377^{* *}$ \\
\hline & G & 0.023 & -0.002 & 0.012 & -0.004 & 0.000 & 0.403 & 0.113 & -0.058 & -0.012 & -0.034 & -0.035 & 0.015 & -0.058 & 0.025 & 0.389 \\
\hline \multirow{2}{*}{$\begin{array}{l}\text { Seed iron }(\mathbf{p p m}) \text { at } \\
\text { maturity }\end{array}$} & $\mathrm{P}$ & 0.005 & 0.004 & 0.012 & -0.012 & 0.003 & 0.378 & -0.029 & -0.003 & 0.000 & -0.004 & -0.063 & 0.002 & -0.005 & 0.016 & $0.305^{*}$ \\
\hline & G & 0.015 & 0.016 & 0.039 & -0.050 & 0.009 & 0.461 & -0.036 & -0.015 & 0.003 & -0.007 & -0.171 & 0.007 & -0.025 & 0.063 & 0.310 \\
\hline \multirow{2}{*}{$\begin{array}{l}\text { Seed calcium uptake } \\
\left(\mathrm{Kg} \mathrm{ha}^{-1}\right)\end{array}$} & $P$ & -0.004 & 0.005 & 0.006 & -0.007 & -0.003 & 0.537 & -0.156 & -0.008 & -0.001 & -0.007 & -0.011 & 0.011 & -0.014 & 0.013 & $0.360^{*}$ \\
\hline & G & -0.014 & 0.017 & 0.018 & -0.031 & -0.009 & 0.657 & -0.199 & -0.042 & -0.007 & -0.012 & -0.029 & 0.042 & -0.075 & 0.051 & 0.367 \\
\hline \multirow{2}{*}{$\begin{array}{l}\text { Seed sulphur uptake } \\
\left(\mathrm{Kg} \mathrm{ha}^{-1}\right)\end{array}$} & $\mathrm{P}$ & -0.004 & 0.006 & 0.012 & -0.007 & -0.003 & 0.633 & -0.100 & -0.013 & -0.002 & -0.013 & -0.017 & 0.008 & -0.017 & 0.016 & $0.500^{* *}$ \\
\hline & $\mathrm{G}$ & -0.013 & 0.022 & 0.038 & -0.029 & -0.009 & 0.775 & -0.126 & -0.068 & -0.015 & -0.021 & -0.045 & 0.033 & -0.095 & 0.063 & 0.509 \\
\hline \multirow{2}{*}{$\begin{array}{l}\text { Seed iron uptake } \\
\left(\mathrm{Kg} \mathrm{ha}^{-1}\right)\end{array}$} & $\mathrm{P}$ & -0.003 & 0.007 & 0.016 & -0.014 & -0.001 & 0.655 & -0.118 & -0.009 & -0.001 & -0.006 & -0.048 & 0.007 & -0.013 & 0.021 & $0.494^{* *}$ \\
\hline & $\mathrm{G}$ & -0.011 & 0.028 & 0.052 & -0.060 & -0.002 & 0.800 & -0.150 & -0.046 & -0.008 & -0.010 & -0.130 & 0.026 & -0.071 & 0.083 & 0.501 \\
\hline
\end{tabular}

Phenotypic residual effect: 0.1141, Genotypic residual effect: SQRT (1- 1.0524). Diagonal (Bold) : Direct effect; Non-diagonal (Normal) : Indirect effects 
The characters days to $50 \%$ flowering and days to maturity recorded significant negative association with pod yield per plant. The negative correlation of days to maturity with pod yield per plant indicated that selection for early maturing varieties may be helpful to maintain high yield potential under reduced crop duration with increased yield under rainfed conditions.

The inter correlation among component traits revealed that, kernel yield per plant registered significant positive association with shelling percent, harvest index, seed iron uptake. These results were in agreement with the reports of Shoba et al., (2012) for shelling percent, Kumar et al., (2013) for harvest index. Number of pods per plant displayed significant positive association with seed calcium (\%) at maturity which was earlier reported by Mandal et al., (2005).

Correlation coefficients, though give information regarding the association of different component traits, it does not project the complete picture especially when the causal factors are interrelated, hence path analysis is used as a tool to understand the magnitude and direction of the direct and indirect contribution of component traits to pod yield (Table 2). All those characters which registered significant association with pod yield were subjected to path analysis to access their direct and indirect effects to pod yield. Path co-efficient analysis revealed that kernel yield per plant exerted maximum direct effect on pod yield per plant followed by days to $50 \%$ flowering, plant height, seed iron uptake and seed calcium uptake. These results are in accordance with the reports of Sadeghi and Niyaki (2012) for kernel yield per plant

Conversely, the highest negative direct effect on pod yield per plant was registered by number of pods per plant followed by seed sulphur $(\%)$ at maturity, leaf iron (ppm) at 60 DAS, seed sulphur uptake, days to maturity, harvest index, seed iron (ppm) at maturity, number of branches per plant and shelling percent. Days to 50 per cent flowering exhibited positive indirect effects on pod yield through shelling percent, harvest index, number of pods per plant, leaf iron (ppm) at 60 DAS and seed sulphur uptake.

The present finding of negative effect of number of primary branches per plant on pod yield is in conformity with the earlier reports of Kumar et al., (2014) while present report of negative direct effect of number of primary branches per plant on pod yield is in contrary with the earlier reports of Sumathi and Murlidharan (2007).

From the above discussion, it is evident that kernel yield per plant emerged as major component of pod yield to emphasize in selection process. In the present study, the residual effect was of low magnitude (0.1141), suggesting that the characters included in the present study are nearly sufficient to explain the causal components of genetic variation in pod yield per plant.

Critical analysis of results obtained from character association and path analysis indicated that characters viz., kernel yield per plant, seed iron uptake and seed calcium uptake had strong positive association with pod yield, which also had the high to moderate magnitude of positive direct effects on pod yield.

Moreover, the indirect effects of most of the characters via kernel yield per plant were positive. It is, therefore, suggested that preference should be given to kernel yield per plant, harvest index, seed iron uptake and seed calcium uptake in the selection programme to isolate superior lines with genetic potential for high pod yield and high nutrient uptake traits.

\section{References}

Amarasinghe, Y. P. J., Wijesinghe, G and Pushpakumara R. W. 2016. Multivariate Analysis, Genetic Diversity and Phenotypic Correlation of Nineteen Exotic Groundnut Accessions. Journal of Agrisearch. 3(1): 7-12. 
Anonymous 2015.Annual Report, AICRPGroundnut. Directorate of Groundnut research, Junagarh, Gujrat, India.

Arntez, C. J. 1994. Encyclopaedia of Agricultural Science. Groundnut (Arachis hypogaea L.). Academic press.3:112.

Bhargava, B.S and Raghupati, H.B. 1993. Analysis of Plant Materials for Macro and Micronutrients. In: Methods of Analysis of Soils, Plants, Waters and Fertilizers. Edited by: Tandon, H.L.S. 1993. Fertilizer Development and Consultation Organisation, New Delhi. pp: 144-166.

Dewey, D. R and Lu, K. H. 1959. A correlation and path coefficient analysis of components of Crested wheatgrass seed production. Agronomy Journal. 51: 515518.

Jackson, M.L. 1967. Soil Chemical analysis. Prentice Hall of India (Pvt.) Ltd., New Delhi. p. 114.

Johnson, H. W., Robinson, H. F and Comstock, R. E. 1955b. Genotypic and phenotypic correlation in soybean and their implications in selection. Agronomy Journal. 47: 477-483.

Kumar, C. P., Rekha, R., Venkateswarulu, O and Vasanthi, R. P. 2013. Correlation and Path Coefficient Analysis in Groundnut (Arachis hypogaea L.). International Journal of Applied Biology and Pharmaceutical Technology. 5(1): 8-11.

Kumar, K., Rai, P. K., Kumar, A., Singh B. A and Chaurasia A. K. 2014. Study on the performance of groundnut (Arachis hypogea L.) genotypes for Quantitative Traits in Allahabad region. Caribbean Journal of Science and Technology. 2. 564-569.

Lindsay, W.L and Norvell, W.A. 1978. Development of DTPA Soil test for Zinc, Iron, Copper and Manganese. Soil
Science Society of American Journal. 42: 421:428.

Mandal, S., Samui, R.C and Anirban Mandal. 2005. Growth, yield and yield attributes of groundnut (Arachis hypogaea L.) cultivar as influenced by gypsum application. Legume Research. 28(2): 119-121.

Nadaf, H.L., Habib, A.F and Goud, J.V. 1986. Analysis of genetic diversity in bunch groundnut. Journal of Oil Seeds Research. 3: 37-45.

Ramesh, G., 2006. Relationship between different forms of soil sulphur and leaf sulphur contents and yield of groundnut. Indian Journal of Dry-land Agricultural Research and Development. 21: 27- 30.

Sadeghi, S.M. and Niyaki, S.A.N. 2012. Correlation and path coefficient analysis in peanut (Arachis hypogaea L) genotypes under drought stress and irrigated conditions. Annals of Biological Research. 3 (6): 2593-2596.

Sharma, L. K and Gupta, S. C. Nature and Magnitude of Association of Pod Yield with Different Morphological Characters in Parents and Hybrids of Groundnut (Arachis hypogaea L.). 2008. National Journal of Plant Improvement. 10(2): 129-132.

Shoba, D., Manivannan, N and Vindhiyavarman, P. 2012. Correlation and Path Coefficient Analysis in Groundnut (Arachis hypogaea L.). Madras Agricultural Journal. 99 (1-3): 18-20.

Sumathi, P and Muralidharan, V. 2007. Character association and path coefficient analysis in confectionery type groundnut (Arachis hypogaea L.). Madras Agricultural Journal. 94 (1-6): 105-109.

\section{How to cite this article:}

Mohammad Raza, M. Reddi Sekhar, M. Shanthi Priya, K.V. Nagamadhuri and Rajarajeswari, V. 2018. Character Association and Path Analysis of Pod Yield and Nutrient Uptake Traits in Groundnut (Arachis hypogaea L.). Int.J.Curr.Microbiol.App.Sci. 7(03): 1570-1577. doi: https://doi.org/10.20546/ijcmas.2018.703.188 